LPT Orsay, 05-48

PCCF RI 0504

\title{
Lattice renormalization of the static quark derivative operator
}

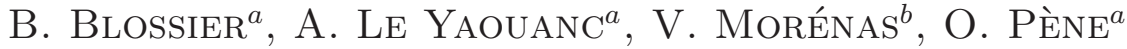 \\ a Laboratoire de Physique Théorique (Bât.210), Université Paris XI-Sud, \\ Centre d'Orsay, 91405 Orsay-Cedex, France. \\ ${ }^{b}$ Laboratoire de Physique Corpusculaire \\ Université Blaise Pascal - CNRS/IN2P3 F-63000 Aubière Cedex, France.
}

\begin{abstract}
We give the analytical expressions and the numerical values of radiative corrections to the covariant derivative operator on the static quark line, used for the lattice calculation of the Isgur-Wise form factors $\tau_{1 / 2}(1)$ and $\tau_{3 / 2}(1)$. These corrections induce an enhancement of renormalized quantities if an hypercubic blocking procedure is used for the Wilson line, while there is a reduction without such a procedure.
\end{abstract}

PACS: 12.38.Gc (Lattice QCD calculations), 12.39.Hg (Heavy quark effective theory), 13.20.He (Leptonic/semileptonic decays of bottom mesons). 


\section{Introduction}

In a previous paper [1] we proposed a method to compute on the lattice, in the static limit of HQET, the Isgur-Wise form factors $\tau_{1 / 2}(1)$ and $\tau_{3 / 2}(1)$ which parameterize decays of $B$ mesons into orbitally excited (P wave) $D^{* *}$ charmed mesons. Keep in mind that the zero recoil is the only definite limit of HQET on the lattice, because the Euclidean effective theory with a non vanishing spatial momentum of the heavy quark is not lower bounded. Then it reveals impossible to calculate directly the $\tau_{j}$ 's from the currents because the matrix elements vanish at zero recoil. To compute $\tau_{1 / 2}(1)$ and $\tau_{3 / 2}(1)$, we proposed to evaluate on the lattice the matrix elements $\left\langle H_{0}^{*}(v)\left|\bar{h}(v) \gamma_{i} \gamma_{5} D_{j} h(v)\right| H(v)\right\rangle$ and $\left\langle H_{2}^{*}(v)\left|\bar{h}(v) \gamma_{i} \gamma_{5} D_{j} h(v)\right| H(v)\right\rangle$, using the following equalities:

$$
\begin{gathered}
\left\langle H_{0}^{*}(v)\left|\bar{h}(v) \gamma_{i} \gamma_{5} D_{j} h(v)\right| H(v)\right\rangle=i g_{i j}\left(M_{H_{0}^{*}}-M_{H}\right) \tau_{\frac{1}{2}}(1), \\
\left\langle H_{2}^{*}(v)\left|\bar{h}(v) \gamma_{i} \gamma_{5} D_{j} h(v)\right| H(v)\right\rangle=-i \sqrt{3}\left(M_{H_{2}^{*}}-M_{H}\right) \tau_{\frac{3}{2}}(1) \epsilon_{i j}^{*},
\end{gathered}
$$

where $D_{i}$ is the covariant derivative $\left(D_{i}=\partial_{i}+i g A_{i}\right), M_{H}, M_{H_{0}^{*}}$ and $M_{H_{2}^{*}}$ are the mass of the $0^{-}, 0^{+}$and $2^{+}$states respectively, and $\epsilon_{i j}^{*}$ is the polarization tensor. These relations are defined between renormalized quantities. Then we have to renormalize the matrix element of the derivative operator computed on the lattice. We explained that power and logarithmic divergences are not to be feared in the zero recoil limit. However finite renormalization is present and we want to establish the one-loop contributions to the derivative operator with the hypercubic blocking [2] of the Wilson line.

We have to renormalize and to match onto the continuum the bare operator $O_{i j}^{B}=$ $\bar{h}^{B} \gamma_{i} \gamma^{5} D_{j} h^{B}$, where $h^{B}$ is the bare heavy quark field. We choose the MOM scheme whose renormalization conditions are: 1) the renormalized heavy quark propagator is equal to the free one, and 2) the renormalized vertex function taken between renormalized external legs is the tree level one.

The rhs of equations (1) and (2) are independent of the renormalization scale $\mu$. Indeed, on the one hand, $M_{H_{0}^{*}}-M_{H} \equiv \bar{\Lambda}_{0^{+}}-\bar{\Lambda}_{0^{-}}$and $M_{H_{2}^{*}}-M_{H} \equiv \bar{\Lambda}_{2^{+}}-\bar{\Lambda}_{0^{-}}$are physical quantities. On the other hand,

$$
\begin{aligned}
\frac{\left\langle D_{0}\left|\bar{c} \gamma^{\mu} \gamma^{5} b\right| B\right\rangle}{\sqrt{m_{B} m_{D_{0}}}} & \equiv g^{+}\left(v+v^{\prime}\right)^{\mu}+g^{-}\left(v-v^{\prime}\right)^{\mu} \\
& \equiv-\tau_{1 / 2}(\mu, w) \sqrt{w-1} F^{\mu}, \\
F^{\mu} & =\sqrt{w+1} C_{1}^{5}(\mu, w) a^{\mu}+\sqrt{w-1}\left[C_{2}^{5}(\mu, w) v^{\mu}+C_{3}^{5}(\mu, w) v^{\prime \mu}\right],
\end{aligned}
$$

where $\sqrt{w^{2}-1} a^{\mu}=\left(v-v^{\prime}\right)^{\mu}$, and the $C_{i}^{5}$ 's are the matching coefficients between the QCD operator $\bar{c} \gamma^{\mu} \gamma^{5} b$ and the HQET operators $\bar{c}_{v^{\prime}} \gamma^{\mu} \gamma^{5} b_{v}, \bar{c}_{v^{\prime}} v^{\mu} \gamma^{5} b_{v}$ and $\bar{c}_{v^{\prime}} v^{\prime \mu} \gamma^{5} b_{v}$ respectively.

$$
\begin{array}{rll}
\frac{\left\langle D_{0}\left|\bar{c} \gamma^{\mu} \gamma^{5} b\right| B\right\rangle}{\sqrt{w-1} \sqrt{m_{B} m_{D_{0}}}} & = & -\tau_{1 / 2}(\mu, w) F^{\mu} \\
& \longrightarrow w \rightarrow 1 & -\tau_{1 / 2}(\mu, 1) \sqrt{2} C_{1}^{5}(\mu, 1) a^{\mu}
\end{array}
$$


$C_{1}^{5}(\mu, 1) \equiv C_{1}^{5}(1)[3]\left(C_{1}^{5}(1) \equiv \eta_{A}=0.986 \pm 0.005\right.$ [4] $)$ and the lhs of (3) is also independent of $\mu$ : thus $\tau_{1 / 2}(\mu, 1) \equiv \tau_{1 / 2}(1)$. We can use the same argument to prove the scale independence of $\tau_{3 / 2}(\mu, 1)$. Consequently the scale $\mu$ will be omitted in the following.

It is well known that the heavy quark self-energy diverges linearly in $1 / a[5]$, so we introduce a mass counterterm $\delta m$ to cancel this divergence. Numerically it is canceled nonperturbatively in the ratio between the three-point function and the two-point functions to obtain a matrix element, or in the difference between binding energies of heavy-light mesons.

The bare heavy propagator on the lattice is

$$
\begin{aligned}
S^{B}(p) & =\frac{a}{1-e^{-i p_{4} a}+a \delta m+a \Sigma(p)} \\
& =\frac{a}{1-e^{-i p_{4} a}} \sum_{n}\left(\frac{-a[\delta m+\Sigma(p)]}{1-e^{-i p_{4} a}}\right)^{n} \\
& \equiv Z_{2 h} S^{R}(p) .
\end{aligned}
$$

By choosing the renormalization conditions

$$
\left.\left(S^{R}\right)^{-1}(p)\right|_{i p_{4} \rightarrow 0}=i p_{4}, \quad \delta m=-\Sigma\left(p_{4}=0\right),
$$

the constant $Z_{2 h}$ is then:

$$
Z_{2 h}=1-\left.\frac{d \Sigma}{d\left(i p_{4}\right)}\right|_{i p_{4} \rightarrow 0} .
$$

The bare vertex function $V_{i j}^{B}(p)$ is defined as:

$$
\begin{aligned}
V_{i j}^{B}(p) & =\left(S^{B}\right)^{-1}(p) \sum_{x, y} e^{i p \cdot(x-y)}\left\langle h^{B}(x) O_{i j}^{B}(0) \bar{h}^{B}(y)\right\rangle\left(S^{B}\right)^{-1}(p) \\
& =\frac{Z_{\mathcal{D}}}{Z_{2 h}}\left(S^{R}\right)^{-1}(p) \sum_{x, y} e^{i p \cdot(x-y)}\left\langle h^{R}(x) O_{i j}^{R}(0) \bar{h}^{R}(y)\right\rangle\left(S^{R}\right)^{-1}(p),
\end{aligned}
$$

where

$$
O_{i j}^{B}(0)=Z_{\mathcal{D}} O_{i j}^{R}(0) .
$$

We will see below that $V_{i j}^{B}(p)$ can be written as

$$
\begin{aligned}
V_{i j}^{B}(p) & =(1+\delta V) \bar{u}(p) \gamma_{i} \gamma^{5} p_{j} u(p) \\
& \equiv(1+\delta V) V_{i j}^{R}(p)
\end{aligned}
$$

$\delta V$ is given by all the 1PI one-loop diagrams containing the vertex.

We obtain $\left\langle H^{* *}\left|O_{i j}^{R}\right| H\right\rangle=Z_{\mathcal{D}}^{-1}\left\langle H^{* *}\left|O_{i j}^{B}\right| H\right\rangle$ where $Z_{\mathcal{D}}=Z_{2 h}(1+\delta V)$ and $\left\langle H^{* *}\left|O_{i j}^{B}\right| H\right\rangle$ was computed on the lattice.

This paper is organized as follows: in Sec. 2 we recall the action we use for the heavy quark, we give the corresponding Feynman rules and we clarify our notations; in Sec. 3 we give the analytical expression for the heavy quark self-energy, in Sec. 4 we give the analytical expression for radiative corrections to the derivative operator in lattice HQET. We briefly conclude in Sec. 5 . 


\section{Heavy quark action and Feynman rules}

The lattice HQET action for the static heavy quark is

$$
S^{\mathrm{HQET}}=a^{3} \sum_{n}\left\{h^{\dagger}(n)\left[h(n)-U_{4}^{\dagger, \mathrm{HYP}}(n-\hat{4}) h(n-\hat{4})\right]+a \delta m h^{\dagger}(n) h(n)\right\}
$$

where $U_{4}^{\mathrm{HYP}}(n)$ is a link built from an hypercubic blocking.

We will use in the rest of the paper the following notations taken from [6]-[8]:

$$
\begin{gathered}
\int_{p} \equiv \int_{-\frac{\pi}{a}}^{\frac{\pi}{a}} \frac{d^{4} p}{(2 \pi)^{4}}, \quad \int_{\vec{p}} \equiv \int_{-\frac{\pi}{a}}^{\frac{\pi}{a}} \frac{d^{3} p}{(2 \pi)^{3}}, \quad a^{4} \sum_{n} e^{i p n}=\delta(p), \\
\int_{k} \equiv \int_{-\pi}^{\pi} \frac{d^{4} k}{(2 \pi)^{4}}, \quad \int_{\vec{k}} \equiv \int_{-\pi}^{\pi} \frac{d^{3} k}{(2 \pi)^{3}}, \\
h(n)=\int_{p} e^{i p n} h(p), \\
U_{\mu}(n)=e^{i a g_{0} A_{\mu}^{a}(n) T^{a}}=1+i a g_{0} A_{\mu}^{a}(n) T^{a}-\frac{a^{2} g_{0}^{2}}{2 !} A_{\mu}^{a}(n) A_{\mu}^{b}(n) T^{a} T^{b}+\mathcal{O}\left(g_{0}^{3}\right), \\
U_{\mu}^{\mathrm{HYP}}(n)=e^{i a g_{0} B_{\mu}^{a}(n) T^{a}}=1+i a g_{0} B_{\mu}^{a}(n) T^{a}-\frac{a^{2} g^{2}}{2 !} B_{\mu}^{a}(n) B_{\mu}^{b}(n) T^{a} T^{b}+\mathcal{O}\left(g_{0}^{3}\right), \\
A_{\mu}^{a}(n)=\int_{p} e^{i p\left(n+\frac{a}{2}\right)} A_{\mu}^{a}(p), \quad B_{\mu}^{a}(n)=\int_{p}^{i p\left(n+\frac{a}{2}\right)} B_{\mu}^{a}(p), \\
c_{\mu}=\cos \left(\frac{a\left(p+p^{\prime}\right)_{\mu}}{2}\right), \quad s_{\mu}=\sin \left(\frac{a\left(p+k_{\lambda}^{\prime}\right)}{2}\right) \\
M_{\mu}=\cos \left(\frac{k_{\mu}}{2}\right), \quad N_{\mu}=\sin \left(\frac{k_{\mu}}{2}\right) \\
W=2 \sum_{\lambda} \sin ^{2}\left(\frac{k_{\lambda}}{2}\right)
\end{gathered}
$$

In the Fourier space, the action is given at the order of $\mathcal{O}\left(g_{0}^{2}\right)$ by:

$$
\begin{aligned}
S^{\mathrm{HQET}} & =\int_{p} a^{-1} h^{\dagger}(p)\left(1-e^{-i p_{4} a}\right) h(p)+\delta m h^{\dagger}(p) h(p) \\
& +i g_{0} \int_{p} \int_{p^{\prime}} \int_{q} \delta\left(q+p^{\prime}-p\right)\left(h^{\dagger}(p) B_{4}^{a}(q) T^{a} h\left(p^{\prime}\right) e^{-i\left(p_{4}+p_{4}^{\prime}\right) \frac{a}{2}}\right. \\
& +\frac{a g_{0}^{2}}{2 !} \int_{p} \int_{p^{\prime}} \int_{q} \int_{r} \delta\left(q+r+p^{\prime}-p\right) h^{\dagger}(p) B_{4}^{a}(q) B_{4}^{b}(r) T^{a} T^{b} h\left(p^{\prime}\right) e^{-i\left(p_{4}+p_{4}^{\prime}\right) \frac{a}{2}} .
\end{aligned}
$$

The block gauge fields $B_{\mu}^{a}$ can be expressed in terms of the usual gauge fields: 


$$
B_{\mu}=\sum_{n=1}^{\infty} B_{\mu}^{(n)},
$$

where $B_{\mu}^{(n)}$ contains $n$ factors of $A$. At NLO, it was shown that we only need $B_{\mu}^{(1)}[9]$ :

$$
\begin{aligned}
& B_{\mu}^{(1)}(k)=\sum_{\nu} h_{\mu \nu}(k) A_{\nu}(k), \\
& h_{\mu \nu}(k)=\delta_{\mu \nu} D_{\mu}(k)+\left(1-\delta_{\mu \nu}\right) G_{\mu \nu}(k), \\
& D_{\mu}(k)=1-d_{1} \sum_{\rho \neq \mu} N_{\rho}^{2}+d_{2} \sum_{\rho<\sigma, \rho, \sigma \neq \mu} N_{\rho}^{2} N_{\sigma}^{2}-d_{3} N_{\rho}^{2} N_{\sigma}^{2} N_{\tau}^{2}, \\
& G_{\mu \nu}(k)=N_{\mu} N_{\nu}\left(d_{1}-d_{2} \frac{N_{\rho}^{2}+N_{\sigma}^{2}}{2}+d_{3} \frac{N_{\rho}^{2} N_{\sigma}^{2}}{3}\right), \\
& d_{1}=(2 / 3) \alpha_{1}\left(1+\alpha_{2}\left(1+\alpha_{3}\right)\right), \quad d_{2}=(4 / 3) \alpha_{1} \alpha_{2}\left(1+2 \alpha_{3}\right), \quad d_{3}=8 \alpha_{1} \alpha_{2} \alpha_{3} .
\end{aligned}
$$

Two sets of $\alpha_{i}$ 's have been chosen: 1) $\alpha_{1}=0.75, \alpha_{2}=0.6, \alpha_{3}=0.3$ (which has been chosen in our simulation and has been motivated in [2]) and 2) $\alpha_{1}=1.0, \alpha_{2}=1.0, \alpha_{3}=0.5$, motivated in [10]. We will label these two sets respectively by HYP1 and HYP2.

The Feynman rules can be easily deduced (they must be completed by the application of $\left.h_{\mu \nu}\right)$ :

\begin{tabular}{|c|c|}
\hline heavy quark propagator & $a\left(1-e^{-i p_{4} a}+\epsilon\right)^{-1}$ \\
vertex $V_{\mu, h h g}^{a}\left(p, p^{\prime}\right)$ & $-i g_{0} T^{a} \delta_{\mu 4} e^{-i\left(p_{4}+p_{4}^{\prime}\right) \frac{a}{2}}$ \\
vertex $V_{\mu \nu, h h g g}^{a b}\left(p, p^{\prime}\right)$ & $-\frac{1}{2} a g_{0}^{2} \delta_{\mu 4}\left\{T^{a}, T^{b}\right\} e^{-i\left(p_{4}+p_{4}^{\prime}\right) \frac{a}{2}}$ \\
gluon propagator in the Feynman gauge & $a^{2} \delta_{\mu \nu} \delta^{a b}\left(2 W+a^{2} \lambda^{2}\right)^{-1}$ \\
\hline
\end{tabular}

Note that $p^{\prime}$ and $p$ are the in-going and the out-going fermion momenta, respectively. We also introduce an infrared regulator $\lambda$ for the gluon propagator. We symmetrize the vertex $V_{\mu \nu, h h g g}^{a b}$ by introducing the anti-commutator of the $S U(3)$ generators, normalized by a factor $\frac{1}{2}$. The gluon propagator and the vertices are defined with the $A$ field. The coefficient $\sum_{i=1}^{3} h_{4 i}^{2} \equiv H\left(N_{4}\right)$ will enter as a global multiplicative factor in all the integrals expressed below. We have chosen the Feynman gauge: since one calculates the renormalization of a gauge-invariant operator, the renormalization factor $Z_{\mathcal{D}}$ is gauge invariant.

\section{$3 \quad$ Heavy quark self-energy}

From (4) we have $\Sigma(p)=-\left(F_{1}+F_{2}\right)$, where $F_{1}$ and $F_{2}$ correspond to the diagrams shown in Fig. 1(a) and (b): 


$$
\begin{aligned}
F_{1} & =-\frac{4}{3 a} g_{0}^{2} \int_{k} \frac{H\left(N_{4}\right)}{2 W+a^{2} \lambda^{2}} \frac{e^{-i\left(k_{4}+2 a p_{4}\right)}}{1-e^{-i\left(k_{4}+a p_{4}\right)}+\epsilon} \\
& =-\frac{1}{3 a} g_{0}^{2} \int_{k} \frac{H\left(N_{4}\right)}{N_{4}^{2}+E^{2}} \frac{e^{-i\left(k_{4}+2 a p_{4}\right)}}{1-e^{-i\left(k_{4}+a p_{4}\right)}+\epsilon} \\
& =-\frac{1}{3 a} g_{0}^{2} \int_{k} \frac{H\left(N_{4}\right)}{\left(N_{4}+i E\right)\left(N_{4}-i E\right)} \frac{e^{-i\left(k_{4}+2 a p_{4}\right)}}{1-e^{-i\left(k_{4}+a p_{4}\right)}+\epsilon} \\
& =-\frac{1}{3 a} g_{0}^{2} \int_{\vec{k}} \frac{1}{E} \frac{H(-i E)}{\sqrt{1+E^{2}}} \frac{e^{-2 i a p_{4}}}{e^{E^{\prime}}-e^{-i a p_{4}}} .
\end{aligned}
$$

Note that Latin indices are spatial and

$$
\begin{gathered}
E^{2}=\sum_{i} N_{i}^{2}+\frac{a^{2} \lambda^{2}}{4} \\
H\left(N_{4}\right)=\left(1-d_{1} \sum_{i} N_{i}^{2}+d_{2} \sum_{i<j} N_{i}^{2} N_{j}^{2}-d_{3} N_{1}^{2} N_{2}^{2} N_{3}^{2}\right)^{2} \\
+N_{4}^{2} \sum_{i} N_{i}^{2}\left(d_{1}-\frac{d_{2}}{2} \sum_{j \neq i} N_{j}^{2}+\frac{d_{3}}{3} \prod_{j \neq i} N_{j}^{2}\right)^{2}, \\
E^{\prime}=2 \operatorname{argsh}(E) .
\end{gathered}
$$

In (9) we have eliminated properly the non covariant pole $k_{4}=-a p_{4}+i \epsilon$ by closing the integration contour in the complex plane $\Im\left(k_{4}\right)<0$ where there is the single pole $N_{4}=-i E$. Furthermore the integrals along the lines $k_{4}= \pm \pi+i k_{4}^{\prime}$ are equal, because the integrand is $2 \pi$-periodic.

Finally we have in the limit $a p_{4} \rightarrow 0$ :

$$
\begin{aligned}
& F_{1}=\frac{4}{3 a} g_{0}^{2} \int_{\vec{k}} \frac{H(-i E)}{4 E \sqrt{1+E^{2}}} \frac{1}{1-e^{E^{\prime}}} \\
& +\frac{4}{3} g_{0}^{2} i p_{4} \int_{\vec{k}} \frac{H(-i E)}{2 E \sqrt{1+E^{2}}}\left[\frac{1}{e^{E^{\prime}}-1}+\frac{1}{2} \frac{1}{\left(e^{E^{\prime}}-1\right)^{2}}\right] \text {. }
\end{aligned}
$$
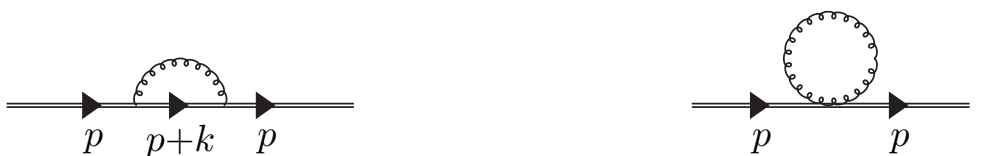

(a): Sunset diagram (b): Tadpole diagram

Figure 1: : Self-energy corrections 
We find:

$$
F_{1} \equiv-\frac{g_{0}^{2}}{12 \pi^{2}}\left\{f_{1}\left(\alpha_{i}\right) / a+i p_{4}\left[2 \ln \left(a^{2} \lambda^{2}\right)+f_{2}\left(\alpha_{i}\right)\right]\right\} .
$$

The tadpole diagram $F_{2}$ is

$$
\begin{aligned}
F_{2} & =-\frac{1}{2} \frac{4 g_{0}^{2}}{3 a} e^{-i a p_{4}} \int_{k} \frac{H\left(N_{4}\right)}{2 W} \\
={ }_{a p_{4} \rightarrow 0} & -\frac{1}{2} \frac{4 g_{0}^{2}}{3}\left(1 / a-i p_{4}\right) \int_{k} \frac{H\left(N_{4}\right)}{2 W} \\
& \equiv \quad-\frac{g_{0}^{2}}{12 \pi^{2}}\left(1 / a-i p_{4}\right) f_{3}\left(\alpha_{i}\right) .
\end{aligned}
$$

The factor $1 / 2$ is introduced to compensate the over-counting of the factor 2 in the Feynman rule of the 2-gluon vertex when a closed gluonic loop is computed. We can point out that the divergent part

$$
\Sigma_{0}\left(\alpha_{i}\right)=\frac{g_{0}^{2}}{12 \pi^{2} a} \sigma_{0}\left(\alpha_{i}\right), \quad \sigma_{0}=f_{1}+f_{3}
$$

of the self-energy is smaller with the sets HYP1 and HYP2 of the $\alpha_{i}$ 's than with the corresponding contribution without "hypercubic" links [5], as shown in the Table 1: $\sigma_{0}\left(\alpha_{i}=\right.$ $0)=19.95, \sigma_{0}(\mathrm{HYP} 1)=5.76$ and $\sigma_{0}(\mathrm{HYP} 2)=4.20$, in good agreement with computations made by the ALPHA collaboration [10], which compares the pseudoscalar heavy-light meson effective energy with different static heavy quark actions, and by Hasenfratz et al [11]. Qualitatively, one expects that the hypercubic blocking reduces the radiative corrections since it amounts roughly to introduce an additional cut-off in the integrals.

The wave function renormalization $Z_{2 h}$ is

$$
Z_{2 h}\left(\alpha_{i}\right)=1+\frac{g_{0}^{2}}{12 \pi^{2}}\left[-2 \ln \left(a^{2} \lambda^{2}\right)+z_{2}\left(\alpha_{i}\right)\right], \quad z_{2}=f_{3}-f_{2} .
$$

$\left|z_{2}\right|$ is also reduced by the hypercubic blocking, as indicated on Table $1: z_{2}\left(\alpha_{i}=0\right)=24.48$ $[5], z_{2}(\mathrm{HYP} 1)=2.52$ and $z_{2}(\mathrm{HYP} 2)=-3.62$.

\section{Derivative operator in lattice HQET}

We have to renormalize the operator $O_{i j}^{B}=\bar{h}^{B} \gamma_{i} \gamma^{5} D_{j} h^{B}$. Following [6],

$$
\begin{aligned}
a^{4} \sum_{n} O_{i j}^{B}(n) & =a^{4} \frac{1}{2 a} \sum_{n} \bar{h}^{B}(n) \gamma_{i} \gamma^{5} U_{j}(n) h^{B}(n+\hat{j})-\bar{h}^{B}(n) \gamma_{i} \gamma^{5} U_{j}^{\dagger}(n-\hat{j}) h^{B}(n-\hat{j}) \\
& =\int_{p} \int_{p}^{\prime} a^{-1} \delta\left(p-p^{\prime}\right) \bar{h}^{B}(p)\left(i \gamma_{i} \gamma^{5} s_{j}\right) h^{B}\left(p^{\prime}\right) \\
& +i g_{0} \int_{p} \int_{p^{\prime}} \int_{q} \delta\left(q+p^{\prime}-p\right) \bar{h}^{B}(p) \gamma_{i} \gamma^{5} c_{i} A_{j}^{a}(q) T^{a} h^{B}\left(p^{\prime}\right) \\
& -\frac{i a g_{0}^{2}}{2 !} \int_{p} \int_{p^{\prime}} \int_{q} \int_{r} \delta\left(q+r+p^{\prime}-p\right) \bar{h}^{B}(p) T^{a} T^{b} \gamma_{i} \gamma^{5} s_{j} A_{j}^{a}(q) A_{j}^{b}(r) h^{B}\left(p^{\prime}\right) .
\end{aligned}
$$


Note that we have chosen to not submit the covariant derivative to the hypercubic blocking.

The vertex function $V_{i j}^{B}$ is obtained by writing $V_{i j}^{B}=V_{i j}^{0}+V_{i j}^{1}+V_{i j}^{2}$, corresponding to the diagrams (a), (b) and (c) in Fig. 2; $V_{i j}^{k}\left(\alpha_{i}\right)=\bar{u}(p) \gamma_{i} \gamma^{5} u(p) V_{j}^{k}\left(\alpha_{i}\right), k=0,1,2$. The contribution $V_{i j}^{0}$ is then given by computing

$$
\begin{aligned}
V_{j}^{0}\left(\alpha_{i}\right) & =-\frac{4 i}{3 a} g_{0}^{2} \int_{k} \frac{H\left(N_{4}\right)}{2 W+a^{2} \lambda^{2}} \sin (k+a p)_{j} \frac{e^{-i\left(k_{4}+2 a p_{4}\right)}}{\left(1-e^{-i\left(k_{4}+a p_{4}\right)}+\epsilon\right)^{2}} \\
& =-\frac{4 i}{3 a} g_{0}^{2} \int_{k} \frac{H\left(N_{4}\right)}{2 W+a^{2} \lambda^{2}}\left(\Gamma_{j}+a p_{j} \cos k_{j}\right) e^{-i a p_{4}}\left(\frac{e^{-i \frac{k_{4}+a p_{4}}{2}}}{1-e^{-i\left(k_{4}+a p_{4}\right)}+\epsilon}\right)^{2} \\
& =-\frac{4 i}{3 a} g_{0}^{2} \int_{k} \frac{H\left(N_{4}\right)}{2 W+a^{2} \lambda^{2}}\left(\Gamma_{j}+a p_{j} \cos k_{j}\right)\left(1-i a p_{4}\right) \frac{1}{\left[2 i \sin \left(\frac{k_{4}+a p_{4}}{2}\right)+e^{i \frac{k_{4}+a p_{4}}{2}} \epsilon\right]^{2}} .
\end{aligned}
$$

We can get rid of the integrand proportional to $\Gamma_{j}$, because it is an odd term. It remains

$$
V_{j}^{0}\left(\alpha_{i}\right)=-\frac{4}{3} i g_{0}^{2} p_{j} \int_{k} \frac{H\left(N_{4}\right)}{2 W+a^{2} \lambda^{2}} \frac{\cos k_{j}}{\left(2 i N_{4}+\epsilon M_{4}\right)^{2}} .
$$

The integrand has poles at $N_{4}= \pm i E, k_{4}=2 i \operatorname{argth}\left(\frac{\epsilon}{2}\right)$. Once again we close the integration contour around the single pole $N_{4}=-i E$. Since $j$ is spatial, the "sail" diagram drawn on Fig. 2(b) does not give any contribution, thus $V_{i j}^{1}=0$. There is finally the tadpole contribution $V_{i j}^{2}$ which is given by computing

$$
V_{j}^{2}\left(\alpha_{i}\right)=-\frac{1}{2 !} \frac{4}{3} i g_{0}^{2} p_{j} \int_{k} \frac{1}{2 W}=-\frac{i g_{0}^{2}}{12 \pi^{2}} p_{j} 12.23 .
$$

We have finally $\left\langle H^{* *}\left|O_{i j}^{R}\right| H\right\rangle=Z_{\mathcal{D}}^{-1}\left(\alpha_{i}\right)\left\langle H^{* *}\left|O_{i j}^{B}\right| H\right\rangle\left(\alpha_{i}\right)$ where

$$
\begin{aligned}
Z_{\mathcal{D}}\left(\alpha_{i}\right) & =Z_{2 h}\left(\alpha_{i}\right)\left[1+\delta V\left(\alpha_{i}\right)\right], \\
\delta V\left(\alpha_{i}\right) & =-\frac{4 g_{0}^{2}}{3} \int_{k}\left(\frac{H\left(N_{4}\right) \cos k_{j}}{\left(2 W+a^{2} \lambda^{2}\right)\left(2 i N_{4}+\epsilon M_{4}\right)^{2}}+\frac{1}{4 W}\right) \\
& =-\frac{4 g_{0}^{2}}{3} \frac{1}{2} \int_{\vec{k}} \frac{H(-i E) \cos k_{j}}{(2 E)^{3} \sqrt{1+E^{2}}}-\frac{g_{0}^{2}}{12 \pi^{2}} 12.23 \\
& \equiv \frac{g_{0}^{2}}{12 \pi^{2}}\left[2 \ln \left(a^{2} \lambda^{2}\right)+f_{4}\left(\alpha_{i}\right)\right],
\end{aligned}
$$
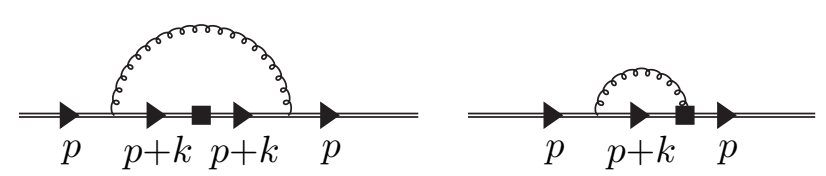

(a) (b)

Figure 2: : Operator corrections 


\begin{tabular}{|c|c|c|c|}
\hline & $\alpha_{i}=0$ & HYP1 & HYP2 \\
\hline$f_{1}$ & 7.72 & 1.64 & -1.76 \\
$f_{2}$ & $-12,25$ & 1.60 & 9.58 \\
$f_{3}$ & 12.23 & 4.12 & 5.96 \\
$f_{4}$ & -12.68 & -10.32 & -8.18 \\
\hline$\sigma_{0}$ & 19.95 & 5.76 & 4.20 \\
$z_{2}$ & 24.48 & 2.52 & -3.62 \\
$z_{d}$ & 11.80 & -7.80 & -11.80 \\
\hline
\end{tabular}

Table 1: Numerical values of the parameters $f_{1}, f_{2}, f_{3}, f_{4}, \sigma_{0}, z_{2}, z_{d}$ defined in equations (11), (12), (18), (13), (14) and (19), respectively.

$$
Z_{\mathcal{D}}\left(\alpha_{i}\right)=1+\frac{g_{0}^{2}}{12 \pi^{2}} z_{d}\left(\alpha_{i}\right), \quad z_{d}=z_{2}+f_{4} .
$$

The numerical values of $z_{d}$ are indicated in Table 1 .

Remark that infrared divergences appearing in $Z_{2 h}$ and $1+\delta V$ cancel and there is no dependence on $a$, a further consequence of the $\mu$ independence of the matrix element $\left\langle H_{0}^{*}\left(v^{\prime}\right)\left|\bar{h}\left(v^{\prime}\right) \gamma_{i} \gamma_{5} D_{j} h(v)\right| H(v)\right\rangle$ at zero recoil. Note also that as already mentioned the tadpole diagram of the operator vertex is not smoothed by the hypercubic blocking; therefore it is quite large. On the other hand the tadpole contribution to the self-energy is smoothed by the blocking. The final result is a large positive correction to the renormalized matrix element. By fixing $g_{0}=1(\beta=6.0)$, this gives $Z_{D}^{-1}(\mathrm{HYP} 1)=1.07$ and $Z_{D}^{-1}(\mathrm{HYP} 2)=1.10$, thus the discrepancy between $Z_{D}^{-1}(\mathrm{HYP} 1)$ and $Z_{D}^{-1}(\mathrm{HYP} 2)$ is small $(3 \%)$; without hypercubic blocking one would obtain $Z_{D}^{-1}\left(\alpha_{i}=0\right)=0.90$. We can think of applying a boosting procedure; in the pure HYP case the boosting plaquette correction is very small [8] (equation 19 and below). On the other hand we have to take into account that the covariant derivative operator involves links without hypercubic blocking, therefore one should employ a different prescription for the diagram drawn on Fig. 2(c), possibly leading to a larger tadpole contribution from the operator to $Z_{D}^{-1}\left(\alpha_{i} \neq 0\right)$, and therefore a larger positive correction. Anyhow this kind of recipe would not lead to $Z_{D}^{-1}\left(\alpha_{i} \neq 0\right)$ significantly larger than 1.1.

With our exploratory lattice study and taking account $Z_{\mathcal{D}}^{-1}$ (HYP1), we find $\tau_{\frac{1}{2}}(1)=$ $0.41(5)(?), \tau_{\frac{3}{2}}(1)=0.57(10)(?)$ and $\tau_{\frac{3}{2}}^{2}(1)-\tau_{\frac{1}{2}}^{2}(1)=0.15(10)$, where systematics are unknown; one is then not too far (within $1 \sigma$ ) from saturating by ground states the Uraltsev sum rule [12] $\sum_{n}\left|\tau_{\frac{3}{2}}^{(n)}(1)\right|^{2}-\left|\tau_{\frac{1}{2}}^{(n)}(1)\right|^{2}=\frac{1}{4}$. However the relation $\mu_{\pi}^{2}-\mu_{G}^{2}>9 \Delta^{2} \tau_{\frac{1}{2}}^{2}(1)$ [13], with $\Delta \equiv M_{H_{0}^{*}}-M_{H}=0.4 \mathrm{GeV}$ [1], $\mu_{G}^{2}=0.35 \mathrm{GeV}^{2}$, leads to $\mu_{\pi}^{2}$ larger than 0.6 $\mathrm{GeV}^{2}$, which is significantly above experimental determination by moments.

\section{Conclusion}

In this paper we have calculated the radiative corrections to the covariant derivative operator $\bar{h} \gamma_{i} \gamma^{5} D_{j} h$ in lattice HQET with an hypercubic blocking of the Wilson line defining 
the heavy quark propagator. This determines the renormalization of the operator which is used to estimate the Isgur-Wise functions between the ground state and the $L=1$ excitations at zero recoil. We find that there is a global, but moderate, enhancement of $\tau_{\frac{1}{2}}(1)$ and $\tau_{\frac{3}{2}}(1)$ with respect to the bare quantities computed on the lattice in the case where one introduces fat timelike links, while there is a reduction with a simple Wilson line.

\section{References}

[1] D. Bećirević et al, Phys. Lett. B 609, 298 (2005)

[2] A. Hasenfratz and F. Knechtli, Phys. Rev. D 64, 034504 (2001), [hep-lat/0103029].

[3] A.V. Manohar and M.B. Wise, "Heavy Quark Physics", Ed. T. Ericson and P.V. Landshoff (Cambridge Monographs on Particle Physics, Nuclear Physics and Cosmology, 2000), p 96

[4] M. Neubert, Phys. Rep. 245, 259 (1994)

[5] E. Eichten and B. Hill, Phys. Lett. B 240, 193 (1990)

[6] S. Capitani, Phys. Rep. 382, 113 (2003), [hep-lat/0222036].

[7] T. DeGrand, Phys. Rev. D 67, 014507 (2003), [hep-lat/0210028].

[8] W. Lee and S. Sharpe, Phys. Rev. D 68, 054510 (2003), [hep-lat/0306016].

[9] W. Lee, Phys. Rev. D 66, 114504 (2002), [hep-lat/0208032].

[10] M. Della Morte, A. Shindler and R. Sommer [ALPHA Collaboration], JHEP 0508, 51 (2005), [hep-lat/0506008]

[11] A. Hasenfratz, R. Hoffmann and F. Knechtli, Nucl. Phys. Proc Suppl 106, 418 (2001), [hep-lat/0110168].

[12] N. Uraltsev, Phys. Lett. B 501, 86 (2001), [hep-ph/0011124].

[13] N. Uraltsev, in Boris Ioffe Festschrift" At the frontier of Particle Physics - Handbook of QCD", Ed. M. Shifman (World Scientific, Singapore, 2001), Vol.3, p. 1577 [hep$\mathrm{ph} / 0010328]$. 